\title{
The completeness of chest X-ray procedure codes in the Danish National Patient Registry
}

\author{
Peter Hjertholm' \\ Kaare Rud Flarup' \\ Louise Mahncke \\ Guldbrandt ${ }^{\prime}$ \\ Peter Vedsted ${ }^{1,2}$ \\ 'Research Center for Cancer \\ Diagnosis in Primary Care, \\ Department of Public Health, \\ ${ }^{2}$ University Clinic for Innovative \\ Health Care Delivery, Diagnostic \\ Centre, Silkeborg Hospital, \\ Department of Clinical Medicine, \\ Aarhus University, Aarhus, Denmark
}

Correspondence: Peter Hjertholm Research Center for Cancer Diganosis in Primary Care, Department of Public Health, Aarhus University, Bartholins Alle 2, 8000 Aarhus, Denmark

Tel +45 404I 4454

Email hjertholm@ph.au.dk
This article was published in the following Dove Press journal:

Clinical Epidemiology

6 March 2017

Number of times this article has been viewed

Objective: The aim of this validation study was to assess the completeness of the registrations of chest X-rays (CXR) in two different versions of the Danish National Patient Registry (DNPR).

Material and methods: We included electronic record data on CXR performed on patients aged 40 to 99 years from nine radiology departments covering 20 Danish hospitals. From each department, we included data from three randomly selected weeks between 2004 and 2011 (reference standard). In two versions of the DNPR from the State Serum Institute (SSI) and Statistics Denmark, respectively, we investigated the proportion of registered CXR compared to the reference standard. Furthermore, we compared the completeness of the recorded data according to the responsible department (main department).

Results: We identified 11,235 patients and 12,513 CXR in the reference standard. The data from the SSI contained 12,265 (98\%) CXR, whereas the data from Statistics Denmark comprised 9,151 (73.1\%) CXR. The completeness of the SSI data was fairly constant across years, radiology departments, medical specialties, and age groups. The data from Statistics Denmark was almost complete in 2011 (95.8\%). However, for the remaining study period, the data with radiology departments registered as the main department were lacking in the version from Statistics Denmark, and so the overall completeness was $73.1 \%$.

Conclusion: The completeness of CXR registrations varied between $98 \%$ and $73 \%$ depending on the information source, and this should be considered when investigating radiology services in the basis of DNPR.

Keywords: chest X-ray, Danish National Patient Registry, diagnostic procedures, validation study, general practice, primary health care

\section{Introduction}

Chest X-ray (CXR) is a key diagnostic tool in the health care system. It is used in the evaluation of acute patients with respiratory problems and chronic disorders and in the diagnostic process of serious diseases. In Denmark, more than 600,000 CXRs are performed each year, and it is thus the most requested radiologic procedure. ${ }^{1} \mathrm{We}$ know that about $20 \%$ of lung cancer patients have had a false-negative CXR prior to diagnosis. $^{2,3}$ Therefore, it is essential to obtain more knowledge about the use of CXR in order to optimize the diagnostic process for different diseases, including cancer.

Registry-based epidemiologic research, based on secondary data, has the advantages of large sample sizes, high statistical precision, and limited risk of recall and nonresponse bias. On the other hand, this type of research greatly depends on the validity and completeness of the data. Studies using registry data are vulnerable (eg, to missing data and selection bias), often with no possibility to discover such problems. 
The Danish National Patient Registry (DNPR) was established in 1977 as a nationwide registry for patient contacts to hospitals. The recorded data is based on the unique civil registration number assigned to all Danish citizens at birth. ${ }^{4}$ Registrations were adapted to the International Classification of Diseases, version 10 (ICD-10) in 1994. DNPR has formed the basis for remuneration of services provided by public hospitals since 2000. It has been mandatory since 2002 for all Danish Hospitals to report all performed radiological procedures to the DNPR; this also includes registration of all CXRs performed.

All Danish radiology departments use a version of an electronic registration system called the Radiology Information System (RIS). The version may vary between hospitals, but all systems ensure that a valid civil registration number is registered before an X-ray can be conducted. Therefore, this system is considered to contain complete and valid information on all radiology procedures performed. Data from the RIS are not readily accessible to researchers, but the RIS provides the basis for the information in the DNPR. ${ }^{5}$ The DNPR was hosted by the National Board of Health until March 2012 after which the State Serum Institute (SSI), the Danish National Institute for Health and Disease Control, was given the responsibility. The data are processed regionally through different algorithms before being transferred to the SSI. Data are accessible for researchers either directly from the SSI or through Statistics Denmark which receives copies from SSI on an annual basis. Statistics Denmark receives the data for national statistical figures, but the organization also hosts data for researchers.

Only two previous studies have investigated the completeness of the registrations in DNPR of performed diagnostic procedures (coronary computed tomographic [CT] angiography and fetal umbilical artery flow velocity); both of these studies showed low completeness. ${ }^{6,7}$

The aim of this study was to investigate the completeness of the CXR registrations in the DNPR by comparing the two versions from the SSI and Statistics Denmark, respectively, using RIS as the reference standard.

\section{Methods}

\section{Design}

We conducted a validation study comparing DNPR data from two different sources (the SSI and Statistics Denmark) and used the RIS data to examine the completeness of the performed CXR coding.

\section{Data}

\section{RIS - the reference standard}

We invited the administrators of ten different randomly selected radiology departments to provide data for the study. Nine departments agreed to participate. Data from RIS were collected from these nine radiology departments that were located across the nation and covered 20 different hospitals. We established a cohort of all persons with a valid civil registration number who had a CXR performed in one of the included radiology departments between January 1, 2004, and December 31, 2011, and were aged between 40 and 99 years at the date of investigation. From each department, we requested data for three different weeks during the entire study period. The weeks were randomly distributed in the period 2004-2011 using the RAND function in Excel. Aarhus University Hospital did not have data before 2009 and could therefore only provide information on two of the randomly assigned weeks, which gave a total of 29 different weeks of CXR. Using the civil registration number provided to all Danish citizens, we were able to link data at the individual level. We did not have information on the exact time of the day for each CXR. Therefore, we allowed inclusion of only one CXR per person per day.

\section{Statistics Denmark and the SSI}

From the SSI, we had information on all CXRs performed at Danish hospitals in the period 2004-2011 according to the DNPR for persons aged 40-99 years. These data were searched for CXRs performed on our study population at the relevant hospitals. From Statistics Denmark, we obtained annual copies from 2004 to 2011 of the DNPR. We extracted data on our study population regarding performed CXRs at the relevant hospitals (procedure code: UXRC00). In all registrations of diagnostic procedures in both versions of the DNPR, the patient was assigned a main department that was responsible for their procedure and treatment. This is generally the department referring for the procedure. For outpatients with CXR referral from elsewhere, mainly general practice, the radiology department was coded as the main department. The classification of CXR (UXRC00) and other procedures in the DNPR are based on the web-based Health Care Classification (SKS) system. ${ }^{8}$ The SKS system is a collection of international, Nordic, and Danish classifications that contain up to ten alphanumeric characters. The first is a letter representing a primary group, and the records follow a mono-hierarchical classification system. The SKS system covers diagnoses, surgery, other treatments, anesthesia, and examinations. ${ }^{5}$ The code UXRC00 encompasses both frontal and lateral CXR. However, lateral CXR is performed only in patients who can mobilize from the bed. 


\section{Analyses}

Completeness was calculated as the proportion of performed CXR procedures from RIS registered with the SSI or Statistics Denmark, respectively. We also compared the registrations of the main department between the two versions of the DNPR. Analyses were stratified according to age at investigation (40-54, 55-69, 70-84, and 85-99 years) and year of investigation. Mean age was calculated on the basis of the integer of age at the date of the CXR.

All analyses and data management were carried out using the statistical software Stata 14.1 (StataCorp LP, Lakeway Drive, College Station, TX, USA).

\section{Ethics}

The study was approved by the Danish Data Protection Agency (J.no. 2013-41-2606). According to Danish law, approval from the National Committee on Biomedical Research Ethics was not required as no biomedical intervention was performed. Patient written informed consent was deemed not required for this study according to Danish law, due to the use of de-identified register data.

\section{Results}

\section{RIS - the reference standard}

The cohort from RIS consisted of 11,235 persons who had a total of 12,513 CXRs performed during the 29 investigated weeks. Mean age of patients was 66.3 years (Table 1).

\section{SSI}

In the data from the SSI, we were able to link 12,265 (98.0\%) performed CXRs (Table 2) with a mean patient age of 66.3 years (Table 1). A radiology department was the most frequently registered main department (Table 3 ). The completeness of data was comparable across age groups and throughout the years (Table 2). The proportion of missing data was almost equally distributed across the radiology departments (Table 2). Only small differences existed across radiology departments when stratified according to age (Table 4)

\section{Statistics Denmark}

In the version of the DNPR from Statistics Denmark, we were able to link 9,151 CXR (73.1\%) to the reference standard. The proportion of linked CXR varied between the years from $50.9 \%$ in 2005 to $95.8 \%$ in 2011 . The mean age of the patients was 67.5 years (Table 1). Most of the patients had internal medicine as their main department (Table 3). Only $6.9 \%$ had a radiology department as their main department (Table 3); all of these 629 CXR were registered in 2011 (data not shown). Therefore, the main reason for the higher
Table I Characteristics of the population having a CXR performed according to the RIS (reference standard) and matched CXR in the SSI and Statistics Denmark, respectively

\begin{tabular}{|c|c|c|c|}
\hline & RIS & SSI & $\begin{array}{l}\text { Statistics } \\
\text { Denmark }\end{array}$ \\
\hline Number of $X$-rays & 12,513 & 12,265 & 9,151 \\
\hline Number of individual persons & 11,235 & $|I, 00|$ & 8,041 \\
\hline \multicolumn{4}{|l|}{ per person } \\
\hline Age, mean & 66.3 & 66.3 & 67.5 \\
\hline Radiology department & $n(\%)$ & $\mathrm{n}(\%)$ & $\mathrm{n}(\%)$ \\
\hline \multicolumn{4}{|l|}{ Aalborg University Hospital } \\
\hline Aalborg & $\mathrm{I}, 777(\mathrm{I} 4.2)$ & $\mathrm{I}, 776(\mid 4.5)$ & $\mathrm{I}, 494(16.3)$ \\
\hline Farsø, Himmerland & $505(4.0)$ & $504(4.1)$ & $324(3.5)$ \\
\hline Sygehus Vendsyssel & $873(7.0)$ & $873(7.1)$ & $672(7.3)$ \\
\hline \multicolumn{4}{|l|}{ Aarhus University Hospital } \\
\hline Aarhus & $\mathrm{I}, \mathrm{I} 03(8.8)$ & $\mathrm{I}, 035(8.4)$ & $909(9.9)$ \\
\hline Samsø & $8(0.1)$ & $7(0.1)$ & $5(0.1)$ \\
\hline $\begin{array}{l}\text { Viborg Regional Hospital } \\
\text { (including Kjellerup) }\end{array}$ & $649(5.2)$ & $640(5.2)$ & $47 \mid(5.2)$ \\
\hline Skive & $286(2.3)$ & $279(2.3)$ & $199(2.2)$ \\
\hline Esbjerg Hospital & $827(6.6)$ & $826(6.7)$ & $443(4.8)$ \\
\hline \multicolumn{4}{|l|}{ Hospital Lillebaelt } \\
\hline Vejle (including Middelfart) & $646(5.2)$ & $630(5.1)$ & $444(4.9)$ \\
\hline Fredericia & $385(3.1)$ & $376(3.1)$ & $228(2.5)$ \\
\hline Kolding & $427(3.4)$ & $421(3.4)$ & $235(2.6)$ \\
\hline Give & $73(0.6)$ & $73(0.6)$ & $3(0.03)$ \\
\hline Odense University Hospital & $\mathrm{I}, 657(13.2)$ & $1,622(13.2)$ & $1,122(12.3)$ \\
\hline \multicolumn{4}{|l|}{ Zealand University Hospital } \\
\hline Roskilde & 801 (6.4) & 757 (6.2) & $523(5.7)$ \\
\hline Køge & $458(3.7)$ & $433(3.5)$ & $267(2.9)$ \\
\hline Fakse & $50(0.4)$ & $50(0.4)$ & $7(0.1)$ \\
\hline Slagelse Hospital & $463(3.7)$ & $460(3.8)$ & $378(4.1)$ \\
\hline Gentofte Hospital & $1,524(12.2)$ & $1,503(12.3)$ & I,427 (I5.6) \\
\hline
\end{tabular}

Abbreviations: CXR, chest X-ray; RIS, radiology information system; SSI, State Serum Institute.

proportion of missing data in Statistics Denmark was that no CXRs were registered with radiology as the main department before 2011. Some differences in the proportion of missing data existed across radiology departments (Table 2). There was a tendency for a higher completeness among the elderly, both overall (Table 2) and for each hospital (Table 4).

\section{Discussion}

We found that the completeness of the DNPR varied according to the data source. Compared to the data obtained directly from the radiology departments, the version from the SSI had a high completeness; $98 \%$ of all CXRs were registered on the same date. The version from Statistics Denmark had a higher proportion of missing data; this was mainly because no CXR was registered with radiology as the main department before 2011.

All patients registered with a diagnostic procedure in the DNPR are assigned a main department. An algorithm 
Table 2 The completeness of the DNPR from the SSI and Statistics Denmark, respectively, compared to the registrations by radiology departments of I2,5 I 3 CXR (reference standard)

\begin{tabular}{|c|c|c|c|}
\hline & \multicolumn{2}{|l|}{ (reference) } & $\begin{array}{l}\text { Statistics } \\
\text { Denmark }\end{array}$ \\
\hline & n (\%) & n (\%) & n (\%) \\
\hline Overall, $n$ & $12,513(100)$ & $12,265(98.0)$ & $9,15 \mid(73.1)$ \\
\hline \multicolumn{4}{|l|}{ Year of CXR } \\
\hline 2004 & $755(100)$ & $748(99.1)$ & $582(77.1)$ \\
\hline 2005 & $672(100)$ & $656(97.6)$ & $342(50.9)$ \\
\hline 2006 & $1,853(100)$ & I,835 (99.0) & I,395 (75.3) \\
\hline 2007 & $638(100)$ & $631(98.9)$ & $427(66.9)$ \\
\hline 2008 & $2,692(100)$ & $2,658(98.7)$ & $\mathrm{I}, 904(70.7)$ \\
\hline 2009 & $1,065(100)$ & $\mathrm{I}, 046(98.2)$ & $675(63.4)$ \\
\hline 2010 & $2,591(100)$ & $2,502(96.6)$ & $\mathrm{I}, 674(64.6)$ \\
\hline 2011 & $2,247(100)$ & $2,189(97.4)$ & $2,152(95.8)$ \\
\hline \multicolumn{4}{|l|}{ Age, years } \\
\hline $40-54$ & $2,510(100)$ & $2,446(97.5)$ & I,635 (65.I) \\
\hline $55-69$ & $4,716(100)$ & $4,616(97.9)$ & $3,259(69.1)$ \\
\hline $70-84$ & $4,28 I(100)$ & $4,210(98.3)$ & $3,388(79.1)$ \\
\hline $85-99$ & $1,006(100)$ & $993(98.7)$ & $869(86.4)$ \\
\hline \multicolumn{4}{|l|}{ Radiology department } \\
\hline Aalborg University & $3,155(100)$ & $3,153(99.9)$ & $2,490(78.9)$ \\
\hline \multicolumn{4}{|l|}{ Hospital } \\
\hline Aarhus University Hospital & $\mathrm{I}, \mathrm{II}(\mathrm{I}(100)$ & $\mathrm{I}, 042(93.8)$ & $914(82.3)$ \\
\hline Viborg Regional Hospital & $936(100)$ & $919(98.2)$ & $670(71.6)$ \\
\hline Esbjerg Hospital & $827(100)$ & $826(99.9)$ & $443(53.6)$ \\
\hline Hospital Lillebaelt & $1,531(100)$ & $1,500(98.0)$ & $910(59.4)$ \\
\hline Odense University & $1,657(100)$ & $\mathrm{I}, 622(97.9)$ & $\mathrm{I}, \mathrm{I} 22(67.7)$ \\
\hline \multicolumn{4}{|l|}{ Hospital } \\
\hline Zealand University & $1,309(100)$ & $\mathrm{I}, 240(94.7)$ & 797 (60.9) \\
\hline \multicolumn{4}{|l|}{ Hospital } \\
\hline Slagelse Hospital & $463(100)$ & $460(99.3)$ & $378(81.6)$ \\
\hline Gentofte Hospital & $1,524(100)$ & I,503 (98.6) & I,427 (93.6) \\
\hline
\end{tabular}

Abbreviations: CXR, chest X-ray; DNPR, Danish National Patient Registry; RIS, radiology information system; SSI, State Serum Institute.

Table 3 Distribution of the five most frequent specialties registered as the main department responsible for the treatment of the patient at the time of the CXR

\begin{tabular}{lll}
\hline Main department & SSI & Statistics Denmark \\
\cline { 2 - 3 } & $\mathbf{n ~ ( \% )}$ & $\mathbf{n ~ ( \% )}$ \\
\hline Radiology & $3,640(29.7)$ & $629(6.9)$ \\
Internal medicine & $2,658(21.7)$ & $2,640(28.9)$ \\
Pulmonary medicine & $1,112(9.1)$ & $1,080(11.8)$ \\
Cardiology & $829(6.8)$ & $819(9.0)$ \\
Thoracic surgery & $763(6.2)$ & $760(8.3)$ \\
\hline
\end{tabular}

Abbreviations: CXR, chest X-ray; SSI, State Serum Institute.

identifies this department according to whether a patient is hospitalized or receives ongoing outpatient care at a department at the time of the procedure. In the former cases, this department is appointed as the main department. Patients referred from general practice for a diagnostic investigation at a radiology department will be assigned the radiology department as the main department. The data from
Statistics Denmark generally lacked information on these investigations from 2004 to 2010. This corresponds to more than 150,000 missing CXRs each year. The completeness increases with age, which may be because a lower proportion of older patients have their CXR taken after referral from general practice. Furthermore, the completeness varies between hospitals; this indicates differences in the proportion of CXRs requested from general practice at the different hospitals. After personal correspondence with both the SSI and Statistics Denmark, it has been confirmed that, in this period of time, all data on visits at a number of laboratory specialties, including radiology, have been deleted before the yearly copies were sent from the SSI to Statistics Denmark. Therefore, researchers are recommended to ensure that full copies of the registry are retrieved and to use the SSI to explore the use of CXR. The SSI data have a very high completeness. However, it is not complete. There may, for example, be small errors in the coding of the civil registration numbers, or different choices may have been made in the algorithms used before the data were sent to the SSI or other reasons. Overall, however, the completeness is regarded very high.

The Danish population-based registries are generally believed to have a high completeness. However, no previous studies have investigated the completeness and validity of radiology codes in DNPR. Previous studies exploring the validity of the DNPR have mainly focused on specific diagnoses and have shown high completeness and validity often over $90 \%$, with some variation between studies. ${ }^{9-17}$ One study, however, found a positive predictive value of $32 \%$ when looking at Vitamin B12 deficiency anemia. ${ }^{13}$ Only two studies have looked at diagnostic investigations. Nielsen et $\mathrm{al}^{7}$ found a completeness of registrations of coronary CT angiographies of $72 \%$ in the DNPR. In a report from the Danish Health and Medicines Authority from 2003, the completeness of the records of fetal umbilical artery flow velocity in the DNPR was $47 \%$ compared to the medical records. ${ }^{6} \mathrm{~A}$ recent review of all validation studies by Schmidt et $\mathrm{a}^{18}$ also confirmed considerable variation in the validity and completeness across diagnoses and treatments. Together with our study, this emphasizes the importance in checking the data thoroughly and critically; it also stresses the necessity of validity studies when looking at data from the DNPR. Furthermore, our findings underscore the significance of good algorithms and reliable data management at the responsible authorities generating the data for the registries.

Some strengths and limitations need to be mentioned. The unique civil registration number assigned to all Danish citizens 
Table 4 The age-stratified completeness of the DNPR from different hospitals for the SSI and Statistics Denmark, respectively, compared to the registrations of the radiology departments of I2,513 CXR (reference standard)

\begin{tabular}{|c|c|c|c|c|}
\hline Hospital & $\begin{array}{l}\text { Age } 40-54 \text { years } \\
\text { n (\% completeness) }\end{array}$ & $\begin{array}{l}\text { Age } 55-69 \text { years } \\
\text { n (\% completeness) }\end{array}$ & $\begin{array}{l}\text { Age } 70-84 \text { years } \\
\text { n (\% completeness) }\end{array}$ & $\begin{array}{l}\text { Age 85-99 years } \\
\text { n (\% completeness) }\end{array}$ \\
\hline \multicolumn{5}{|l|}{ SSI } \\
\hline \multicolumn{5}{|l|}{ Radiology department } \\
\hline Aalborg University Hospital & $634(100)$ & $1,203(99.8)$ & $\mathrm{I}, 043(100)$ & $273(100)$ \\
\hline Aarhus University Hospital & $215(89.2)$ & $415(94.1)$ & $318(95.8)$ & $94(96.9)$ \\
\hline Viborg Regional Hospital & $159(98.2)$ & $300(98.4)$ & $382(98.0)$ & 78 (98.7) \\
\hline Esbjerg Hospital & $154(99.4)$ & $310(100)$ & $305(100)$ & $57(100)$ \\
\hline Hospital Lillebaelt & $317(96.7)$ & $621(98.6)$ & $466(98.9)$ & $96(94.1)$ \\
\hline Odense University Hospital & $316(98.8)$ & $639(97.1)$ & $559(98.1)$ & $108(99.1)$ \\
\hline Zealand University Hospital & $252(94.7)$ & $478(94.5)$ & $403(94.2)$ & $107(98.2)$ \\
\hline Slagelse Hospital & III (98.2) & $136(99.3)$ & $178(100)$ & $35(100)$ \\
\hline Gentofte Hospital & $288(99.0)$ & $514(98.1)$ & $556(98.6)$ & $145(100)$ \\
\hline \multicolumn{5}{|l|}{ Statistics Denmark } \\
\hline \multicolumn{5}{|l|}{ Radiology department } \\
\hline Aalborg University Hospital & $447(70.5)$ & $924(76.7)$ & $875(83.9)$ & $244(89.4)$ \\
\hline Aarhus University Hospital & $180(74.7)$ & $359(8 I .4)$ & $286(86.1)$ & $89(91.8)$ \\
\hline Viborg Regional Hospital & $120(74.1)$ & $201(65.9)$ & $287(73.6)$ & $62(78.5)$ \\
\hline Esbjerg Hospital & $60(38.7)$ & $144(46.5)$ & $195(63.9)$ & $44(77.2)$ \\
\hline Hospital Lillebaelt & $156(47.6)$ & $349(55.4)$ & $323(68.6)$ & $82(80.4)$ \\
\hline Odense University Hospital & $187(58.4)$ & $436(66.3)$ & $423(74.2)$ & $76(69.7)$ \\
\hline Zealand University Hospital & $133(50.0)$ & $251(49.6)$ & $315(73.6)$ & $98(89.9)$ \\
\hline Slagelse Hospital & $83(73.5)$ & III (8I.0) & $152(85.4)$ & $32(91.4)$ \\
\hline Gentofte Hospital & $269(92.4)$ & $484(92.4)$ & $532(94.3)$ & $142(97.9)$ \\
\hline
\end{tabular}

Abbreviations: CXR, chest X-ray; DNPR, Danish National Patient Registry; SSI, State Serum Institute.

at birth enables linkage of big data sets, but it also ensures complete data from the radiology departments (the reference standard), as the radiologic services cannot be initiated without registration of a valid civil registration number. Inclusion of data from nine administrative departments covering 20 hospitals ensured that data are representative of the entire population. During the study period, some hospitals have changed their picture archive and communications system. However, we could not find any differences in the completeness in the SSI version over time for the different hospitals (data not shown). Throughout the study period, the coding of CXR has formed the basis for reimbursement for services provided, which ensures a high completeness of registrations in the reference standard. The random selection of weeks, including weekends and holidays, was also considered a strength.

A potential limitation of the current study is that we only had information on patients aged between 40 and 99 years. Yet, no major variation in the completeness was found for different age groups in the included patients in the SSI version, and it seems unlikely that this would be different among the younger patients. Furthermore, the studied age group receives more than $85 \%$ of the CXRs performed in Denmark. We only included persons with a valid civil registration number and thus excluded tourists; this is because differences exist in the registration of these patients. It would have been a strength if we had been able to calculate the positive predictive value and the validity of a CXR registration in the DNPR, but this was not possible because valid identification of the relevant CXR in the DNPR was uncertain because of numerous administrative changes at radiology departments and hospitals during the study period. It is also a limitation that we were not able to investigate the validity of other information in the records such as the referring body or whether the investigation was performed as inpatient or outpatient. Nevertheless, this information is not registered systematically and uniformly by the radiology departments.

\section{Conclusion}

The completeness of registrations of CXRs in the DNPR differs according to the source of the data. A high completeness was found in the version obtained directly from the SSI, whereas the completeness was under $75 \%$ in the data obtained from Statistics Denmark. This calls for meticulous assessment of both data and data sources in future studies investigating radiologic procedures on the basis of records in the DNPR.

\section{Acknowledgments}

This study received financial support from the Program for Clinical Research Infrastructure, which was established by the 
Lundbeck Foundation and the Novo Nordisk Foundation and administered by the Danish Regions. Furthermore, the work was supported by the Danish National Research Foundation for Primary Care and the Danish Cancer Society. The funding sources had no role in the design and conduct of the study; the collection, analysis, and interpretation of data; or the preparation, review, or approval of the manuscript. We would also like to thank the participating radiology departments for providing data.

\section{Disclosure}

The authors report no conflicts of interest in this work.

\section{References}

1. The Danish Health Data Authority. Esundhed. [The Danish National Patient Registery] http://www.esundhed.dk/sundhedsregistre/LPR/. Updated 2016. Accessed June 29, 2016.

2. Bjerager M, Palshof T, Dahl R, Vedsted P, Olesen F. Delay in diagnosis of lung cancer in general practice. Br J Gen Pract. 2006;56(532):863-868.

3. Stapley S, Sharp D, Hamilton W. Negative chest X-rays in primary care patients with lung cancer. Br J Gen Pract. 2006;56(529):570-573.

4. Pedersen CB. The Danish Civil Registration System. Scand J Public Health. 2011;39(7 Suppl):22-25.

5. Lynge E, Sandegaard JL, Rebolj M. The Danish National Patient Register. Scand J Public Health. 2011;39(7 Suppl):30-33.

6. Langhoff-Roos J, Rasmussen S. Validering af landspatientregistret (LPR) mhp. obstetrisk forskning og kvalitetssikring. Copenhagen, Denmark; 2003. Report in Danish.

7. Nielsen LH, Norgaard BL, Tilsted HH, et al. The Western Denmark Cardiac Computed Tomography Registry: a review and validation study. Clin Epidemiol. 2014;7:53-64.
8. National Health IT S. Sundhedsvæsenets klassifikations system (SKS). [The Classification System of the Danish Health Services] http:// sundhedsdatastyrelsen.dk/da/rammer-og-retningslinjer/om-klassifikationer/sks-klassifikationer. Updated 2016. Accessed November 14, 2016.

9. Vestergaard M, Obel C, Henriksen TB, et al. The Danish National Hospital Register is a valuable study base for epidemiologic research in febrile seizures. J Clin Epidemiol. 2006;59(1):61-66.

10. Lund JL, Froslev T, Deleuran T, et al. Validity of the Danish National Registry of Patients for chemotherapy reporting among colorectal cancer patients is high. Clin Epidemiol. 2013;5:327-334.

11. Thomsen RW, Lange P, Hellquist B, et al. Validity and underrecording of diagnosis of COPD in the Danish National Patient Registry. Respir Med. 2011;105(7):1063-1068.

12. Vest-Hansen B, Riis AH, Christiansen CF. Registration of acute medical hospital admissions in the Danish National Patient Registry: a validation study. Clin Epidemiol. 2013;5:129-133.

13. Ben Ghezala I, Arendt JF, Erichsen R, et al. Positive predictive value of the diagnosis coding for vitamin B12 deficiency anemia in the Danish National Patient Register. Clin Epidemiol. 2012;4:333-338.

14. Rix TA, Riahi S, Overvad K, Lundbye-Christensen S, Schmidt EB, Joensen AM. Validity of the diagnoses atrial fibrillation and atrial flutter in a Danish Patient Registry. Scand Cardiovasc J. 2012;46(3):149-153.

15. Djurhuus BD, Skytthe A, Faber CE. Validation of the cholesteatoma diagnosis in the Danish National Hospital Register. Dan Med Bull. 2010; 57(10):A4159.

16. Wermuth L, Lassen CF, Himmerslev L, Olsen J, Ritz B. Validation of hospital register-based diagnosis of Parkinson's disease. Dan Med J. 2012; 59(3):A4391.

17. Thygesen SK, Olsen M, Christian FC. Positive predictive value of the infant respiratory distress syndrome diagnosis in the Danish National Patient Registry. Clin Epidemiol. 2013;5:295-298.

18. Schmidt M, Schmidt SAJ, Sandegaard JL, Ehrenstein V, Pedersen L, Sørensen HT. The Danish National Patient Registry: a review of content, data quality, and research potential. Clin Epidemiol. 2015;7:449-490.
Clinical Epidemiology

Publish your work in this journal

Clinical Epidemiology is an international, peer-reviewed, open access, online journal focusing on disease and drug epidemiology, identification of risk factors and screening procedures to develop optimal preventative initiatives and programs. Specific topics include: diagnosis, prognosis, treatment, screening, prevention, risk factor modification,

\section{Dovepress}

systematic reviews, risk and safety of medical interventions, epidemiology and biostatistical methods, and evaluation of guidelines, translational medicine, health policies and economic evaluations. The manuscript management system is completely online and includes a very quick and fair peer-review system, which is all easy to use. 